\title{
Effects of the different-sized external stents on vein graft intimal hyperplasia and inflammation
}

\author{
Qi Yang ${ }^{1}$, Dong Lei ${ }^{2}$, Shixing Huang ${ }^{1}$, Yi Yang ${ }^{1}$, Yang Yang ${ }^{3}$, Xiaofeng Ye ${ }^{1}$, Zhengwei You ${ }^{2}$, Qiang Zhao ${ }^{1}$ \\ ${ }^{1}$ Department of Cardiac Surgery, Ruijin Hospital, Shanghai Jiaotong University School of Medicine, Shanghai 200025, China; ${ }^{2}$ State Key Laboratory \\ for Modification of Chemical Fibers and Polymer Materials, Shanghai Belt and Road Joint Laboratory of Advanced Fiber and Low-dimension \\ Materials (Donghua University), College of Materials Science and Engineering, Donghua University, Shanghai 201620, China; ${ }^{3}$ Department of \\ Cardiothoracic Surgery, Xinhua Hospital, Shanghai Jiaotong University School of Medicine, Shanghai 200092, China \\ Contributions: (I) Conception and design: Q Yang, X Ye, Q Zhao; (II) Administrative support: None; (III) Provision of study materials or patients: \\ None; (IV) Collection and assembly of data: Q Yang, D Lei, S Huang; (V) Data analysis and interpretation: Q Yang, S Huang; (VI) Manuscript \\ writing: All authors; (VII) Final approval of manuscript: All authors. \\ Correspondence to: Xiaofeng Ye. Department of Cardiac Surgery, Ruijin Hospital, No. 197, Ruijin Er Road, Huangpu District, Shanghai 200025, \\ China. Email: xiaofengye@hotmail.com; Zhengwei You. State Key Laboratory for Modification of Chemical Fibers and Polymer Materials, Donghua \\ University, No. 2999, North Renmin Road, Songjiang District, Shanghai 201620, China. Email: zyou@dhu.edu.cn; Qiang Zhao. Department of \\ Cardiac Surgery, Ruijin Hospital, No. 197, Ruijin Er Road, Huangpu District, Shanghai 200025, China. Email: zq11607@rjh.com.cn.
}

Background: The poor long-term patency ratio of vein grafts prevents patients from benefiting from coronary artery bypass graft (CABG). It is reported that external venous stents have notably improved the patency ratio of stented vein grafts in animal models. The most crucial influence on stented grafts' fate is the size of the stents. This study aims to investigate the effects on intimal hyperplasia and inflammation of vein graft by using different sizes of stents and explore the potential mechanism.

Methods: Two different sizes of external stents were fabricated through 3D printing technology. Male SD rats were divided into three groups. In the control group rat's autologous left jugular vein was grafted on the ipsilateral artery directly. In the stent groups, grafts were surrounded by two different-sized stents before anastomosing with arteries. The patency ratio and diameter of the grafts were examined by ultrasound. Masson staining was used to characterize intimal hyperplasia. The expression of inflammatory factors was detected by immunohistochemical staining. Moreover, TUNEL staining was used to label apoptotic cells.

Results: The two sizes of external stents were fabricated by $3 \mathrm{D}$ printing technology. In the control group, the intima area and wall thickness dramatically increased 8 weeks after implantation. While in the stent groups, these data only slightly increased, especially in the $1.5 \mathrm{~mm}$-stent group. The expressions of inflammatory factors in TNF signaling were more remarkable than in the control group. On the contrary, the expressions were rarely detected in the stent groups. Similarly, the number of TUNEL positive cells dramatically decreased by using the appropriate-sized stent.

Conclusions: In this study, we concluded that the appropriate sizes of external stents could effectively inhibit vein graft neointima formation, attenuate inflammatory reaction and reduce cell apoptosis, which might improve the long-term patency ratio of vein grafts.

Keywords: Coronary artery bypass graft (CABG); saphenous vein graft; external stent; intimal hyperplasia

Submitted Oct 28, 2019. Accepted for publication Dec 06, 2019.

doi: $10.21037 /$ atm.2020.01.16

View this article at: http://dx.doi.org/10.21037/atm.2020.01.16 


\section{Introduction}

Coronary artery bypass graft (CABG) surgery is the most commonly performed surgical therapy, which is used for relieving angina and improving survival in patients with coronary artery disease (CAD) $(1,2)$. In choosing the bypass conduits, autologous saphenous vein grafts (SVGs) still remain the most frequently used conduits in CABG. However, the lower long-term patency ratio of SVG is the main obstacle to improving treatment efficiency and patients' quality of life (3-6). The reasons for vein graft disease remain numerous. Commonly, acute graft thrombosis due to mechanical injuries, uncontrolled intimal hyperplasia and progressive atherosclerosis caused by several systemic risk factors are responsible for early and late vein graft failure post-implantation $(7,8)$. Besides, SVG expressed less bioactive factor such as NO, tissue plasminogen activator and (t-PA) phosphatase and tensinhomolog (PTEN) compared to arterial bypass grafts, which made SVG vulnerable to detrimental factors (9). Several strategies adopted to avoid SVGs failure, such as medical antiplatelet and aggressive lipid-lowering therapies, or surgical no-touch vein-harvesting technique (10). However, no significant improvement in SVGs failure is observed, indicating that an optimal solution remains to be found.

The use of external stents has shown several significant effects on extending the lifespan of SVGs by reducing vein graft dilation and stabilizing hemodynamics. After seeing several promising results in animal studies (11-14), more and more researchers are concentrating on the therapy of using external stents on patients who receive CABG. However, the studies on humans presented contradictory results (15-20). Besides stent-fabricating materials, which are usually polymers or alloys, another important factor influencing stent efficacy is the diameter of an external stent. Both oversized and over-constricted stents can lead to vein graft failure due to unstable hemodynamic force and vessel injuries $(16,21,22)$.

When vein grafts are exposed to suddenly-increased blood pressure in the arterial circulation, the vessel wall of the vein grafts suffers from severe damage due to mechanical distension and turbulent blood flow shear stress $(23,24)$. This process activates endothelium and stimulate smooth muscle cells (SMCs), which results in inflammatory chemokines expression, inflammatory cell invasion, and vascular remodeling (25-27). Among abundant secreted inflammatory factors, tumor necrosis factor (TNF) is found in vein grafts and plays a vital role in vein graft diseases. The activation of TNF signaling pathway is associated with promoting vascular SMC proliferation and endothelium apoptosis $(28,29)$. Under the composite effects of intima injury and inflammatory response, intimal hyperplasia gradually increased, thus bring about luminal stenosis or even occlusion.

In this study, we fabricated two sizes of external stents and applied them in rats' jugular vein grafts implantation to assess intimal hyperplasia and inflammatory factors expression under different constrictive conditions. Our results indicated that limiting vein graft dilation by using a proper size of the external stent could avoid vascular injury, inhibit intimal hyperplasia and attenuate intima inflammation.

\section{Methods}

\section{Stent fabrication}

The two different sizes of polycaprolactone (PCL) stents were fabricated by $3 \mathrm{D}$ printing technology. Briefly, by using a 3D printer (HTS-400; Fochif Mechatronics Technology, Co., China), the PCL ( $\mathrm{Mn}=80,000 \mathrm{~g} / \mathrm{mol}$, Sigma-Aldrich, USA) solution was squeezed out and deposited on a rotary receiver at a temperature of $180{ }^{\circ} \mathrm{C}$ (Figure $\left.1 A\right)$.

\section{Vein graft procedure}

Vein graft implantation was performed by the 'cuff' technique. Briefly, male Sprague-Dawley (SD) rats weighing 330 to $350 \mathrm{~g}$ were anesthetized with $2 \%$ isoflurane at $4 \mathrm{~L} / \mathrm{min}$ oxygen flow using Isoflurane vaporizer (MATRX VIP 3000). The autologous left jugular vein was carefully dissected and then immersed in heparin saline. The left common carotid artery from the carotid artery bifurcation down to $15 \mathrm{~mm}$ was mobilized and divided. Both ends of the divided artery were everted over self-made cuffs, which were cut from an indwelling needle sheath (20G, BD, Suzhou, China), and ligated with 8-0 prolene sutures. The left jugular vein was anastomosed with the modified artery by inserting the cuff into both ends of the vein graft and ligated the junctions with 8-0 prolene sutures (Ethico, Shanghai, China). After four or eight weeks, animals were sacrificed with intraperitoneal pentobarbitone injection $(75 \mathrm{mg} / \mathrm{kg})$ and the vein grafts were obtained for further studies. 
A
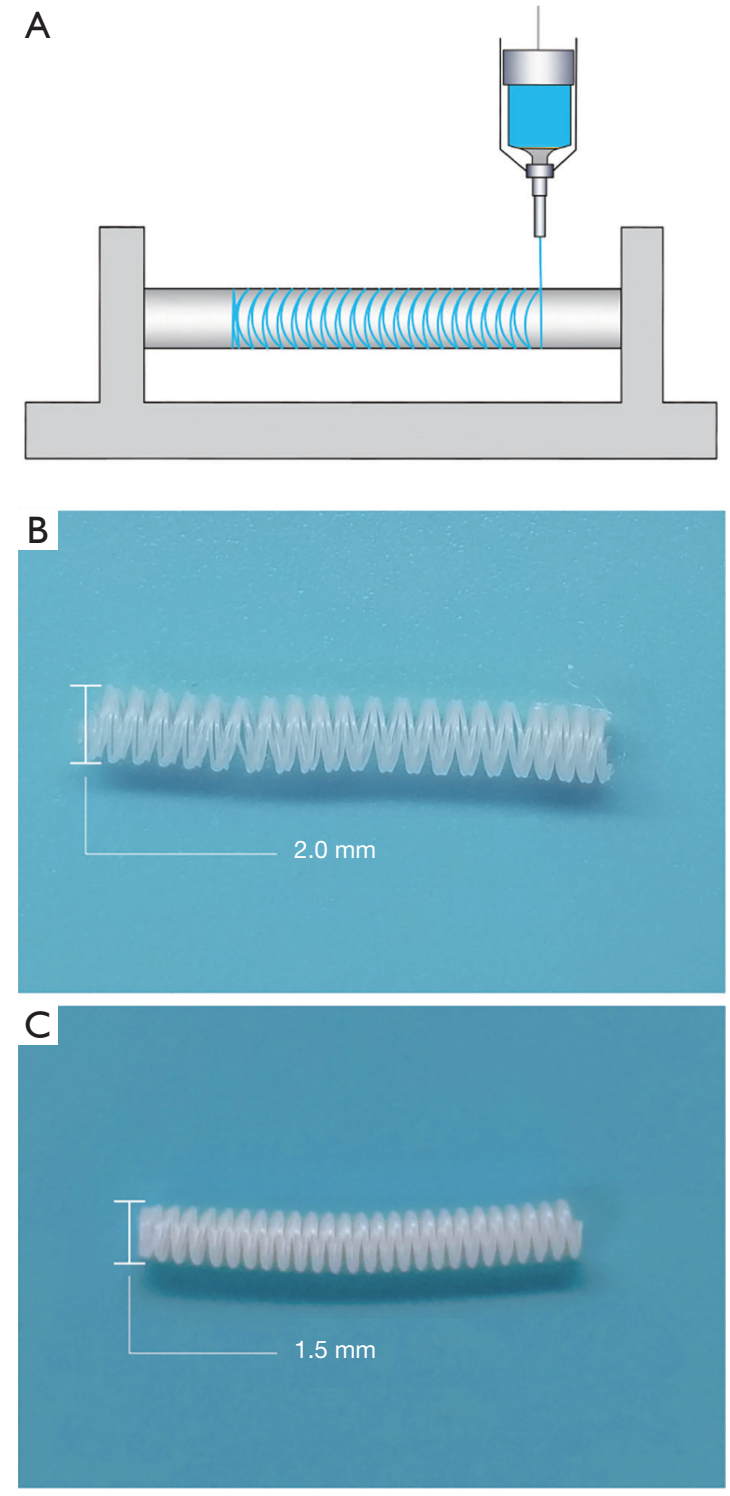

Figure 1 Fabrication and characterization of stents. (A) Schematic illustration of 3D-prined PCL external stent; (B) $2 \mathrm{~mm}$ diameter PCL stent; (C) $1.5 \mathrm{~mm}$-diameter PCL stent. PCL, polycaprolactone.

\section{Vascular ultrasound}

Four or eight weeks after implantation, the rats were anesthetized with isoflurane vapor as previously described. The patency ratio, lumen diameter, velocity of blood flow and the pulse wave were analyzed by a Vevo 2100 ultrasound platform (Visual Sonics, Canada) equipped with a $21 \mathrm{MHz}$ and a $16 \mathrm{MHz}$ probe in evaluation of the condition of the vein grafts.

\section{Histological and immunohistochemical analyses}

Rat vein grafts were fixed with $4 \%$ paraformaldehyde and embedded in paraffin. Samples were sectioned into $5-\mu \mathrm{m}$ thick slices followed by Masson trichrome staining in accordance with the standard protocol (G1006; Servicebio). Image J software was used for a quantitative assessment of the wall thickness and intima area.

To characterize the inflammatory reaction of vein grafts at four- or eight-weeks post-implantation, sections were followed by dewaxing and rehydration procedures. Antigens were retrieved with citrate buffer $(\mathrm{pH} 6.0)$ at $90{ }^{\circ} \mathrm{C}$ for $3 \mathrm{~min}$. The endogenous peroxidase activity was blocked using $3 \% \mathrm{H}_{2} \mathrm{O}_{2}$ solution at room temperature for $25 \mathrm{~min}$. Primary antibodies used were: anti-TNF- $\alpha(1: 100$, GB13188-2, Servicebio, Wuhan, China), anti-JNK(1:100, GB13018-1, Servicebio Wuhan, China), and anti-c-Jun (1:200, GB11515,Servicebio, Wuhan, China). After primary antibody incubation, binding was detected with speciesappropriate biotinylated secondary antibodies (G5001, Servicebio, Wuhan, China), incubation with Horseradish Peroxidase (HRP) (GB233303, Servicebio, Wuhan, China) and chromogenic detection with 3,3-diaminobenzidine (DAB, K5007, DAKO, CA, USA). Nuclei were stained with a hematoxylin counterstain (G1004, Servicebio, Wuhan, China). Slices were then dehydrated and mounted. Image analysis was made by using Image-pro Plus (Media Cybernetics, Rockville, MD, USA).

\section{TUNEL staining}

Terminal deoxynucleotidyl transferase-mediated dUTP nick end labeling (TUNEL) assay was utilized to determine cell apoptosis according to the illustrations (number 11684795910; Hoffman-La Roche Ltd., Basel, Switzerland). After deparaffination, vein graft sections were rehydrated through a graded ethanol series and then incubated with proteinase K solution (G1205, Servicebio, Wuhan, China) at room temperature for $30 \mathrm{~min}$. Later on, the sections were incubated with the reaction buffer provided by the manufacturer at $37^{\circ} \mathrm{C}$ for $3-4 \mathrm{~h}$ and further counterstained with DAPI. TUNEL-positive nuclei in the intimal hyperplasia area were selected in 5 random regions 
of interest (200x magnification) using an LSM880 Meta confocal microscope. The number of TUNEL- positive nuclei was recorded by ImageJ software.

\section{Statistical analysis}

Data were analyzed with SPSS v21.0 software (IBM, Armonk, NY, USA). Differences between groups were evaluated by one-way analysis of variance (ANOVA). Results expressed as mean \pm SD. P values less than 0.05 were considered significant.

\section{Results}

Using 3D printing technology, we fabricated two different sized PCL external stents. The inner diameter of the stent is 2.0 or $1.5 \mathrm{~mm}$, respectively (Figure $1 B, C$ ). The vein grafts were wrapped with those sizes of stents in the stent groups, and the non-stented grafts were used as control (Figure 2). The patency ratio of the implanted vein grafts was $76.2 \%$ (control group, $\mathrm{n}=21$ ), 88.8\% (2.0 mm-stent group, $\mathrm{n}=18$ ), and $94.1 \%(1.5 \mathrm{~mm}$-stent group, $\mathrm{n}=17)$ respectively. Occluded vein grafts were excluded from this study. The ultrasound examination indicated that the diameter of vein grafts in the control group was observably larger than the ones in the $2.0 \mathrm{~mm}$-stent group and the $1.5 \mathrm{~mm}$-stent group. The maximum velocity of blood was the slowest in the control group, and the velocity gradually increased in the stent groups with decreasing stent diameter (Figure 2E).

\section{Vein graft intimal hyperplasia alleviated by using the external stent}

Masson staining revealed the vein graft intimal hyperplasia was the most significant in the control group after four- or eight-weeks post-surgery, and the wall thickness and intima area were increasing over time. On the contrary, in the stent groups, suppression of intimal hyperplasia was presented as the thinner wall thickness and smaller intima area, especially in the $1.5 \mathrm{~mm}$-stent group (Figure 3).

\section{External stent inbibits inflammatory reaction in vein graft}

To illustrate the potential mechanism of the neointimainhibited effect on the vein graft of external stent, we used immunohistochemical staining to detect inflammatory factors of the TNF signaling pathway of vein grafts in each group (Figure $4 A, B, C$ ). The expressions of inflammatory factors of TNF- $\alpha$, JNK, and c-Jun in the control group were more evident than the stent groups, while the expressions descended with decreasing stent size in the stent groups (Figure 4D,E,F).

\section{Apoptosis cells are reduced in stented vein graft}

The TUNEL examining kit was used to detect apoptotic cells. In the control group, TUNEL-positive cells were more assembled in the neointimal region. However, in the stent groups, the appearance of apoptotic cells was reduced and was rarely found in the $1.5 \mathrm{~mm}$-stent group (Figure 5). Detailed information about the results were listed in Table S1.

\section{Discussion}

The frequency of vein graft failures hindered patients benefiting from CABG. In contrast to the high patency ratio of arterial conduits, the long-term patency ratio of SVGs is only $50-60 \%$ after $10-15$ years of followup (4). Researches suggested that vein graft failure is the consequence of the coaction of various factors. Acute thrombosis is the predominant reason leading to early vein graft failure, which could be attributed to mechanical injury (both during and after surgery) of vessel wall and mismatch in conduit size $(7,30)$. Neointimal hyperplasia occurs at the anastomotic region as early as one month after surgery, while varying degrees of intimal hyperplasia could be detected in almost all SVGs one year after implantation. Atherosclerosis, as another important reason leading to vein graft failure after 1-year post-implantation, is affected by most systemic risk factors (8). Different factors are associated with time, and all could eventually result in graft occlusion. Although clinicians have made a variety of efforts to improve vein graft patency and to remodel both medically and surgically, SVGs failures still haunt in patients receiving CABG because of the absence of effective and satisfying therapies (10).

After Parsonnet et al. first explored the use of external stents half a century ago (31), researchers have shown several promising effects of external stents on extending the lifespan of vein grafts in animal models $(12-14,32,33)$. The stent was firstly designed to reduce the diameter mismatch between vein graft and the target vessel, and the vein graft dilation due to higher arterial pressure has also been limited. Most importantly, evidence suggests that vein grafts supported by external stents maintain more stable hemodynamic 
Control

A

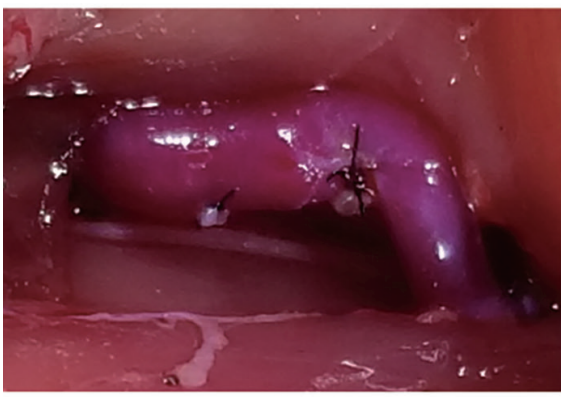

B

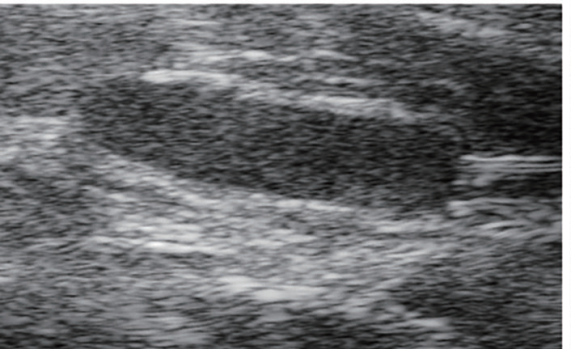

C

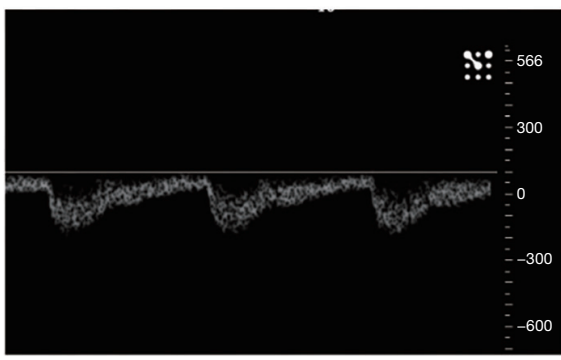

D

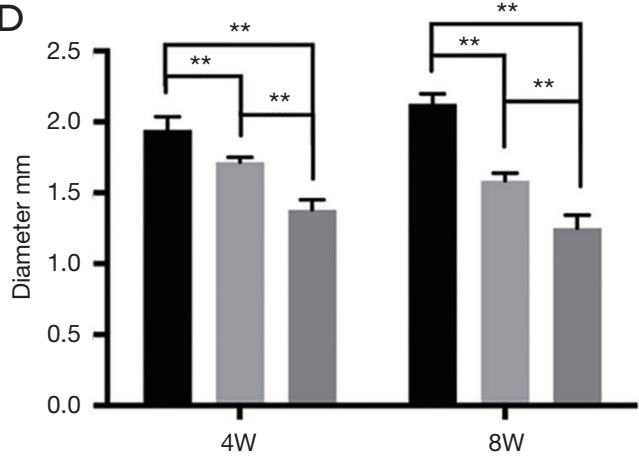

2.0 mm-stent
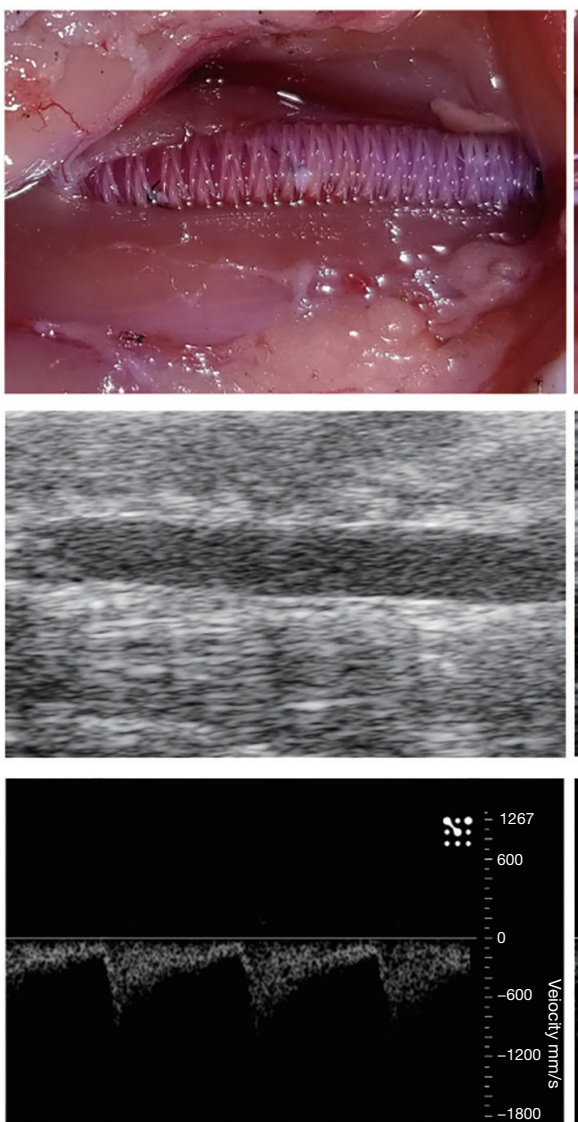

E

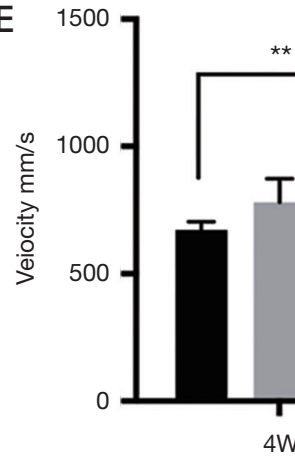

$1.5 \mathrm{~mm}$-stent
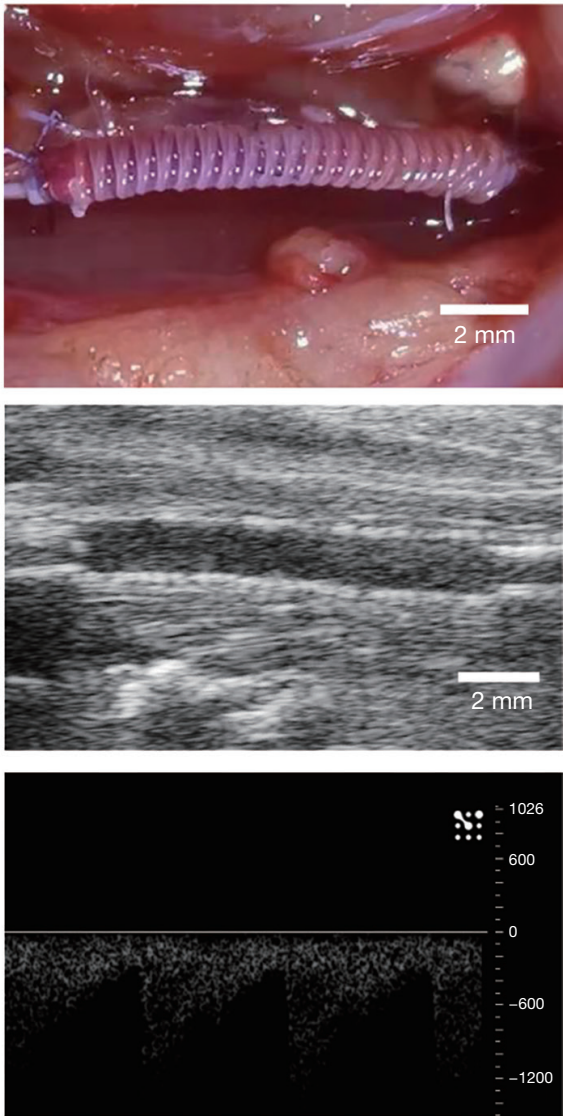

Figure 2 Implantation of vein grafts and vascular ultrasound. (A) implanted vein grafts of control and stent group in vivo; (B,C) image of vascular ultrasound examination; $(\mathrm{D}, \mathrm{E})$ quantification of diameter and velocity of ultrasound. *, $\mathrm{P}<0.05 ;$ **, $\mathrm{P}<0.01$.

flow through reducing blood flow turbulence (34). This effect may protect endothelium and intima from mechanical stretch injury. Totally, in this study, five nonstented grafts and three stented grafts were occluded. In the control group, four grafts were occluded after 4 weeks implantation, which might be cause by the injury of the turbulent blood flow and thrombosis (Figure $2 A$ showed the non-stented graft appeared significant dilation and kink). Meanwhile, all three stented grafts occluded after 8 weeks implantation may resulted from intimal hyperplasia or other factors. These results indicated that the external stents could maintain the straight vascular structure and 
A Control

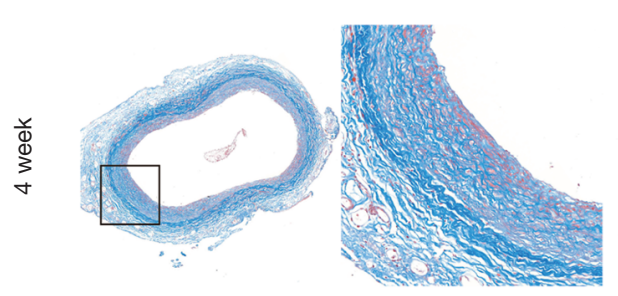

B

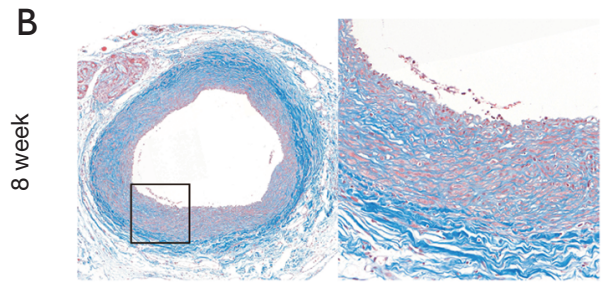

C

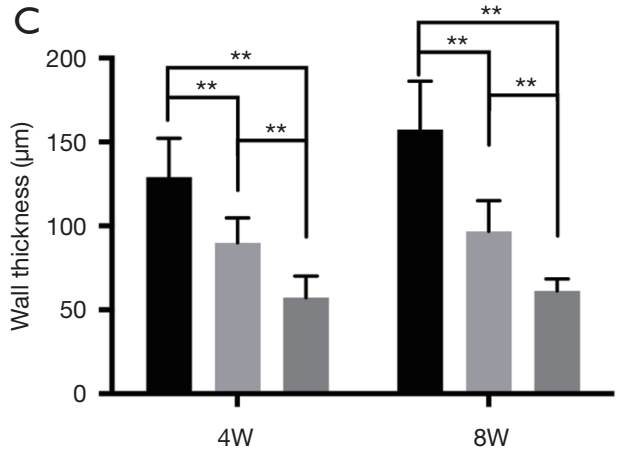

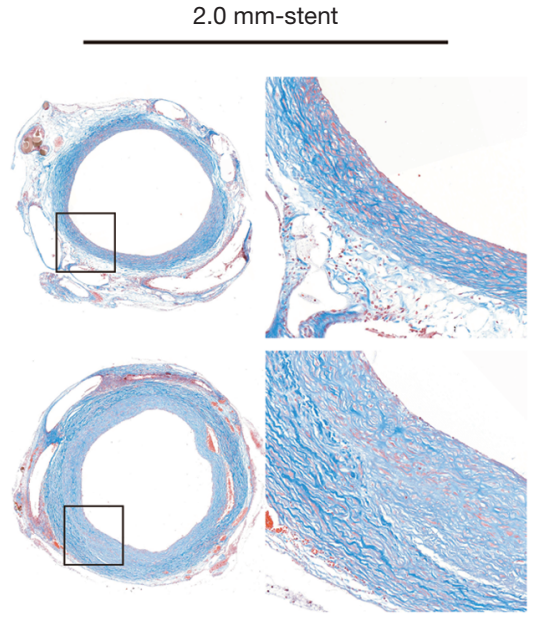
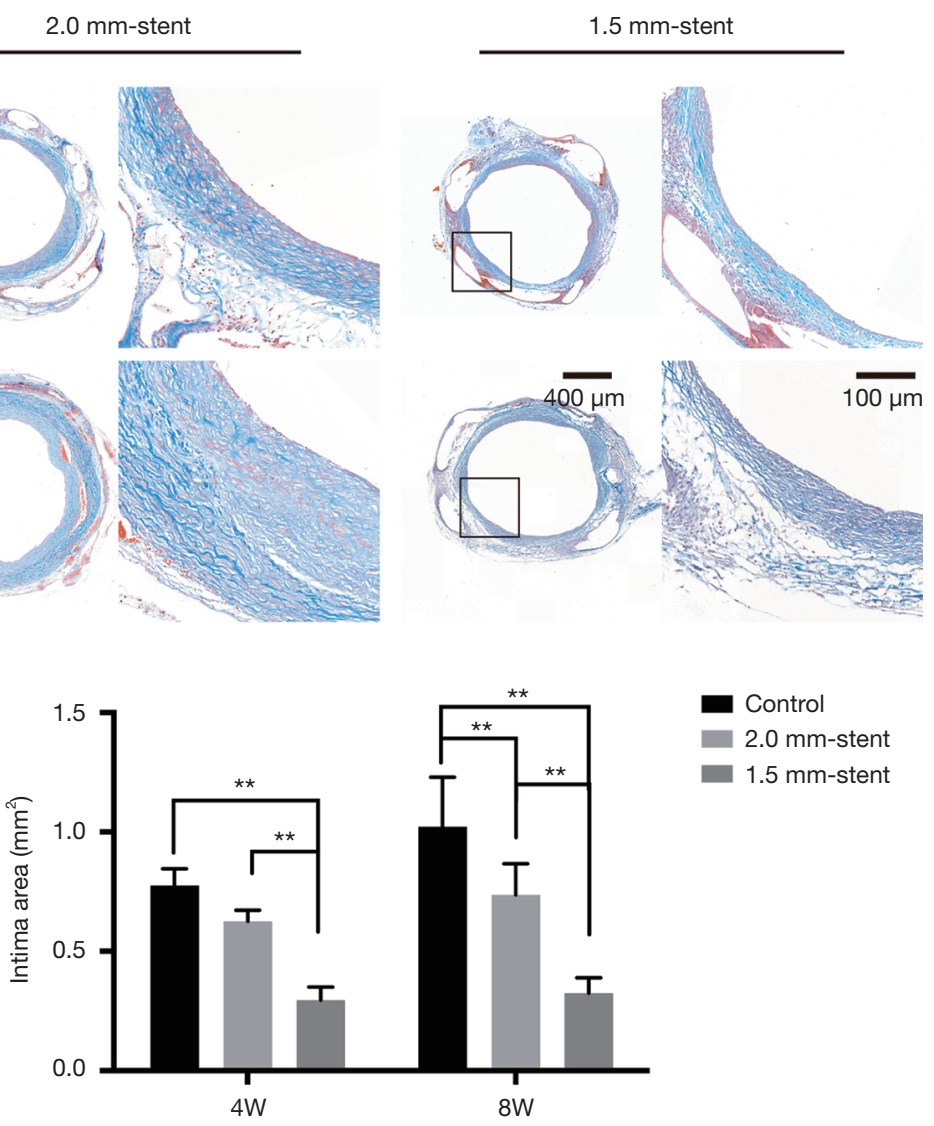

Control $1.5 \mathrm{~mm}$-stent $2.0 \mathrm{~mm}$-stent

Figure 3 Histology characterization of vein graft. (A,B) The Masson staining of vein graft of each groups at 4 or 8 weeks; (C,D) quantification of wall thickness and intima area; ${ }^{* *}, \mathrm{P}<0.01$.

protect vein grafts from being attacked by mechanical injuries, which comes out a better consequence of patency. Interestingly, researches on human studies and clinical trials have demonstrated not only positive but also negative results (35). Barra et al. firstly performed vein support in four patients and shown limited expansion of vein graft (36). While in recent decades, the product of eSVS (Kipsbay Medical Inc., MN, USA) has been studied in a number of randomized trials, which indicated that patency ratio of stented grafts was high in early times but decreased in the long-term observation $(15,17,18,22)$. Studies on VEST (Vascular Graft Solutions LD, Tel Aviv, Israel), the newly designed stent, have shown that the intimal hyperplasia was dramatically reduced in stented grafts, while it had an equal effect on graft patency compared to non-stented grafts $(19,20,37,38)$. Although the results of VEST studies in the short-term were encouraging, whether it can improve graft patency over the long-term is still in need of further studies.

Among all kinds of factors that influence the effect of an external stent, the size of a stent and the stent-fabricating materials are crucial for determining the fate of the vein graft. It is reported that using an oversized stent might result in graft kinking in the middle of the graft as they lack adequate mechanical support, which would bring about disappointing experiences (21). Meanwhile, an overconstricted stent also reduces graft patency due to stenosis and lack of sufficient inflow $(16,22)$. Both situations could lead to an unstable hemodynamic circumstance, which further results in endothelium or intima injury. Human et al. suggests that an average diameter constriction of $27 \%$ might be appropriate to maintain luminal regularities in most of the vein grafts (39). In addition, stent-fabrication materials also determined the stent efficacy. Murphy et al. pointed out that a stiff and undegradable material, such as Dracon, would lead to vein graft failure due to the rigidity and incomplete tube design (21). Subsequent researches of a nitinol alloy stent also showed a disappointing result because the stent needed extra fixation and mismatched with 

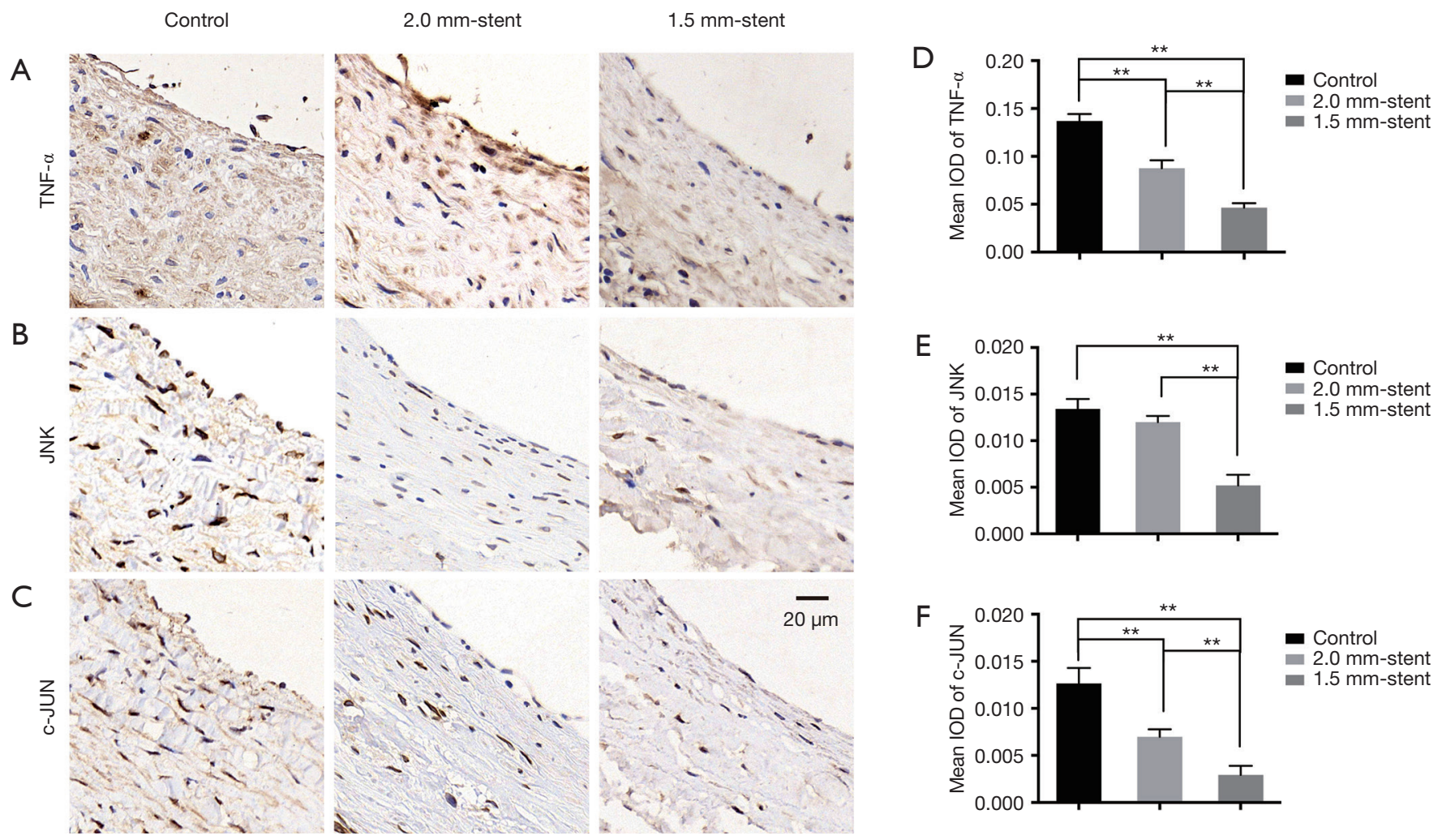

Figure 4 Inflammatory factor expression in each group. (A,B,C) The immunohistochemistry staining of TNF- $\alpha$, JNK and c-Jun in each groups, (D,E,F) quantification of TNF- $\alpha, \mathrm{JNK}$ and c-Jun expressions. ${ }^{* *}, \mathrm{P}<0.01$.

the conduit $(17,22)$. The second generation alloyed stent of VEST (Venous External Scaffolding Technology) consists of the cobalt-chrome braid with plasticity and elasticity, making the device well conform to the conduit (35). The PCL is an FDA-approved polymer which has the advantages of biodegradability and biocompatibility (40). Compared to undegradable alloys or other fast degradable polymers, using such a long-term biodegradable polymer to fabricate stent could provide adequate mechanical support in the early stage after implantation, and avoid foreign body reaction in the late stage after biodegradation (41). In this study, we fabricated two sizes of PCL external stent to adapt to the rats' vein grafts. The $2.0 \mathrm{~mm}$-stent was fit for the diameter of the vein graft when filling with arterial blood, and the $1.5 \mathrm{~mm}$-stent was designed lightly constricted for restricting graft dilation (Figures $1 B, C, 2 A, B$ ). In comparison with the control group, intimal hyperplasia was significantly inhibited in the stent group, especially in the $1.5 \mathrm{~mm}$-stent group (Figure 3), which is consistent with the previous conclusion. These results suggested that the appropriate constriction produced by stent would protect vein graft from suffering intimal hyperplasia. On the contrary, dilation and insufficient restriction of vein graft is more likely appearing luminal restenosis.

The inflammatory processes were involved in all phases during vein graft remodeling. Initial inflammatory response after vein graft implantation is associated with mechanical damage during surgical harvesting and distension due to arterial blood filling. The ischemia-reperfusion during and after surgery generates reactive oxygen species, which stimulates the release of various cytokines and results in vascular wall injury (24). When venous conduits are exposed to arterial pressure, the arterialization process is a positive adaptation based on the proliferation of vascular smooth muscles (SMCs). However, uncontrolled proliferation and migration of SMCs bring about intimal hyperplasia and lumen loss. Those prosses are also accompanied by inflammatory cells infiltrating into the vein graft wall and secreted cytokines and growth factors. It is reported that tumor necrosis factor-alpha (TNF- $\alpha$ ), mainly secreted by macrophages, is involved in vascular inflammation and intimal hyperplasia through inducing adhesion molecule 

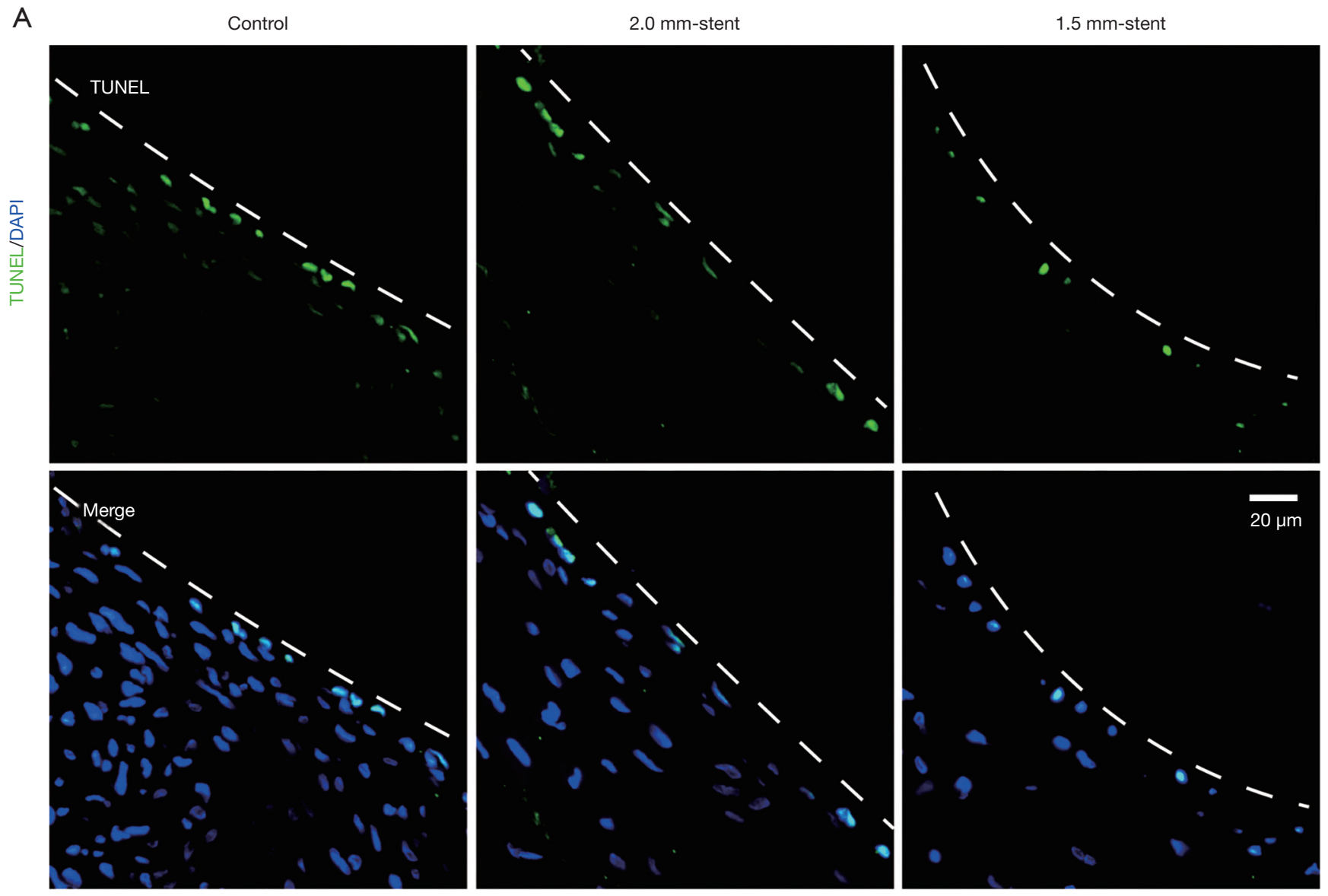

B

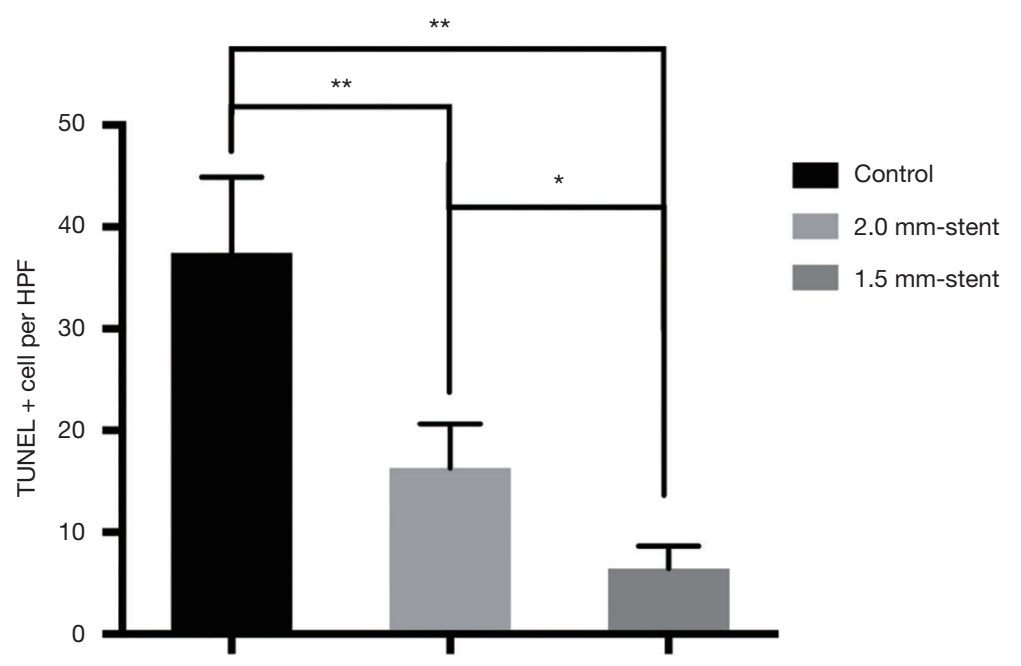

Figure 5 Apoptosis cells of vein grafts in each group. (A) The TUNEL staining labels the apoptosis cells with green fluorescent; (B) quantification of TUNEL positive cells. *, $\mathrm{P}<0.05$; ${ }^{* *}, \mathrm{P}<0.01$. 
in association with cell migration and proliferation $(29,42)$. Moreover, activation of the TNF signaling pathway, including downstream targets such as JNK and c-Jun, is shown to have the effects of promoting cell proliferation (43). In our study, the expressions of inflammatory factors were upregulated in the control group. By using external stents, inflammatory reactions have been attenuated to some extent (Figure 4). This result suggested that the external stents might protect vein graft from inflammatory injury.

In conclusion, by comparing different constrictive conditions, the present study showed that supporting the vein graft with an appropriate size of an external stent could effectively inhibit vein graft neointimal formation, attenuate inflammatory reaction and reduce cells apoptosis. This preclinic research revealed the possible mechanism of the protective effects of external venous stent, which would provide more evidence for clinical treatments in improving the long-term patency ratio of vein graft with external stent.

\section{Acknowledgments}

We gratefully acknowledge the financial supports by the Natural Science Foundation of China (81671832, 81571826 and 21574019), the Natural Science Foundation of Shanghai (18ZR1401900), the Shanghai Municipal Education Commission-Gaofeng Clinical Medicine Grant Support (826158), the Fundamental Research Funds for the Central Universities, DHU Distinguished Young Professor Program (LZA2019001), Shanghai Belt and Road Joint Laboratory of Advanced Fiber and Low-dimension Materials (Donghua University) (18520750400).

\section{Footnote}

Conflicts of Interest: The authors have no conflicts of interest to declare.

Ethical Statement: The authors are accountable for all aspects of the work in ensuring that questions related to the accuracy or integrity of any part of the work are appropriately investigated and resolved. All animals utilized in this study received proper care complied with the National Institutes of Health Guide for the Care and Use of Laboratory Animals (NIH Publications No. 8023, revised 1978), and all surgical operations were approved by the Shanghai Jiaotong University School of Medicine Institutional Animal Care and Use Committee.

\section{References}

1. Yusuf S, Zucker D, Peduzzi P, et al. Effect of coronary artery bypass graft surgery on survival: overview of 10year results from randomised trials by the Coronary Artery Bypass Graft Surgery Trialists Collaboration. Lancet 1994;344:563-70.

2. Eagle KA, Guyton RA, Davidoff R, et al. ACC/AHA 2004 guideline update for coronary artery bypass graft surgery: a report of the American College of Cardiology/ American Heart Association Task Force on Practice Guidelines (Committee to Update the 1999 Guidelines for Coronary Artery Bypass Graft Surgery). Circulation 2004;110:e340-437.

3. Tatoulis J. Total arterial coronary revascularization-patient selection, stenoses, conduits, targets. Ann Cardiothorac Surg 2013;2:499-506.

4. Cao C, Ang SC, Wolak K, et al. A meta-analysis of randomized controlled trials on mid-term angiographic outcomes for radial artery versus saphenous vein in coronary artery bypass graft surgery. Ann Cardiothorac Surg 2013;2:401-7.

5. Yi G, Shine B, Rehman SM, et al. Effect of bilateral internal mammary artery grafts on long-term survival: a meta-analysis approach. Circulation 2014;130:539-45.

6. Mounsey CA, Mawhinney JA, Werner RS, et al. Does Previous Transradial Catheterization Preclude Use of the Radial Artery as a Conduit in Coronary Artery Bypass Surgery? Circulation 2016;134:681-8.

7. Harskamp RE, Lopes RD, Baisden CE, et al. Saphenous vein graft failure after coronary artery bypass surgery: pathophysiology, management, and future directions. Ann Surg 2013;257:824-33.

8. Yahagi K, Kolodgie FD, Otsuka F, et al. Pathophysiology of native coronary, vein graft, and in-stent atherosclerosis. Nat Rev Cardiol 2016;13:79-98.

9. Spadaccio C, Antoniades C, Nenna A, et al. Preventing treatment failures in coronary artery disease: what can we learn from the biology of in-stent restenosis, vein graft failure, and internal thoracic arteries? Cardiovasc Res 2020;116:505-19.

10. Parang P, Arora R. Coronary vein graft disease: pathogenesis and prevention. Can J Cardiol 2009;25:e57-62.

11. Angelini GD, Lloyd C, Bush R, et al. An external, oversized, porous polyester stent reduces vein graft neointima formation, cholesterol concentration, and vascular cell adhesion molecule 1 expression in cholesterol- 
fed pigs. J Thorac Cardiovasc Surg 2002;124:950-6.

12. Jeremy JY, Bulbulia R, Johnson JL, et al. A bioabsorbable (polyglactin), nonrestrictive, external sheath inhibits porcine saphenous vein graft thickening. J Thorac Cardiovasc Surg 2004;127:1766-72.

13. Zilla P, Human P, Wolf M, et al. Constrictive external nitinol meshes inhibit vein graft intimal hyperplasia in nonhuman primates. J Thorac Cardiovasc Surg 2008;136:717-25.

14. Ben-Gal Y, Taggart DP, Williams MR, et al. Expandable external support device to improve Saphenous Vein Graft Patency after CABG. J Cardiothorac Surg 2013;8:122.

15. Schoettler J, Jussli-Melchers J, Grothusen C, et al. Highly flexible nitinol mesh to encase aortocoronary saphenous vein grafts: first clinical experiences and angiographic results nine months postoperatively. Interact Cardiovasc Thorac Surg 2011;13:396-400.

16. Klima U, Elsebay AA, Gantri MR, et al. Computerized tomographic angiography in patients having eSVS Mesh(R) supported coronary saphenous vein grafts: intermediate term results. J Cardiothorac Surg 2014;9:138.

17. Inderbitzin DT, Bremerich J, Matt P, et al. One-year patency control and risk analysis of eSVS(R)-meshsupported coronary saphenous vein grafts. J Cardiothorac Surg 2015;10:108.

18. Rescigno G, Aratari C, Matteucci SM, et al. Saphenous Vein Graft Wrapping by Nitinol Mesh: A Word of Caution. Thorac Cardiovasc Surg 2015;63:292-7.

19. Taggart DP, Ben Gal Y, Lees B, et al. A Randomized Trial of External Stenting for Saphenous Vein Grafts in Coronary Artery Bypass Grafting. Ann Thorac Surg 2015;99:2039-45.

20. Taggart DP, Amin S, Djordjevic J, et al. A prospective study of external stenting of saphenous vein grafts to the right coronary artery: the VEST II study. Eur J Cardiothorac Surg 2017;51:952-8.

21. Murphy GJ, Newby AC, Jeremy JY, et al. A randomized trial of an external Dacron sheath for the prevention of vein graft disease: the Extent study. J Thorac Cardiovasc Surg 2007;134:504-5.

22. Emery RW, Solien E, Klima U. Clinical evaluation of the eSVS Mesh: First-in-Man trial outcomes. ASAIO J 2015;61:178-83.

23. Gaudino M, Antoniades C, Benedetto U, et al. Mechanisms, Consequences, and Prevention of Coronary Graft Failure. Circulation 2017;136:1749-64.

24. de Vries MR, Quax PHA. Inflammation in Vein Graft Disease. Front Cardiovasc Med 2018;5:3.
25. Golledge J, Gosling M, Turner RJ, et al. Arterial flow induces changes in saphenous vein endothelium proteins transduced by cation channels. Eur J Vasc Endovasc Surg 2000;19:545-50.

26. Bonetti PO, Lerman LO, Lerman A. Endothelial dysfunction: a marker of atherosclerotic risk. Arterioscler Thromb Vasc Biol 2003;23:168-75.

27. Ruiter MS, Pesce M. Mechanotransduction in Coronary Vein Graft Disease. Front Cardiovasc Med 2018;5:20.

28. Zhang L, Peppel K, Brian L, et al. Vein graft neointimal hyperplasia is exacerbated by tumor necrosis factor receptor-1 signaling in graft-intrinsic cells. Arterioscler Thromb Vasc Biol 2004;24:2277-83.

29. Wajant H, Pfizenmaier K, Scheurich P. Tumor necrosis factor signaling. Cell Death Differ 2003;10:45-65.

30. Baikoussis NG, Argiriou M, Dedeilias P, et al. External Stenting of the Vein Grafts in Coronary Artery Operations: Limitations, Benefits, and Patients' Safety. Ann Thorac Surg 2015;100:1969.

31. Parsonnet V, Lari AA, Shah IH. New Stent for Support of Veins in Arterial Grafts. Arch Surg 1963;87:696-702.

32. Zilla $P$, Wolf $M$, Rafiee $N$, et al. Utilization of shape memory in external vein-graft meshes allows extreme diameter constriction for suppressing intimal hyperplasia: a non-human primate study. J Vasc Surg 2009;49:1532-42.

33. Sato A, Kawamoto S, Watanabe M, et al. A novel biodegradable external mesh stent improved longterm patency of vein grafts by inhibiting intimal-medial hyperplasia in an experimental canine model. Gen Thorac Cardiovasc Surg 2016;64:1-9.

34. Meirson T, Orion E, Di Mario C, et al. Flow patterns in externally stented saphenous vein grafts and development of intimal hyperplasia. J Thorac Cardiovasc Surg 2015;150:871-8.

35. Mawhinney JA, Mounsey CA, Taggart DP. The potential role of external venous supports in coronary artery bypass graft surgery. Eur J Cardiothorac Surg 2018;53:1127-34.

36. Barra JA, Volant A, Leroy JP, et al. Constrictive perivenous mesh prosthesis for preservation of vein integrity. Experimental results and application for coronary bypass grafting. J Thorac Cardiovasc Surg 1986;92:330-6.

37. Webb CM, Orion E, Taggart DP, et al. OCT imaging of aorto-coronary vein graft pathology modified by external stenting: 1-year post-surgery. Eur Heart J Cardiovasc Imaging 2016;17:1290-5.

38. Taggart DP, Webb CM, Desouza A, et al. Long-term performance of an external stent for saphenous vein grafts: the VEST IV trial. J Cardiothorac Surg 2018;13:117. 
39. Human P, Franz T, Scherman J, et al. Dimensional analysis of human saphenous vein grafts: Implications for external mesh support. J Thorac Cardiovasc Surg 2009;137:1101-8.

40. Nyitray CE, Chang R, Faleo G, et al. Polycaprolactone Thin-Film Micro- and Nanoporous Cell-Encapsulation Devices. ACS Nano 2015;9:5675-82.

41. Yang Y, Lei D, Huang S, et al. Elastic 3D-Printed Hybrid Polymeric Scaffold Improves Cardiac Remodeling

Cite this article as: Yang Q, Lei D, Huang S, Yang Y, Yang Y, Ye X, You Z, Zhao Q. Effects of the different-sized external stents on vein graft intimal hyperplasia and inflammation. Ann Transl Med 2020;8(4):102. doi: 10.21037/atm.2020.01.16 after Myocardial Infarction. Adv Healthc Mater 2019;8:e1900065.

42. Malinska A, Perek B, Buczkowski P, et al. CD68 expression in aortocoronary saphenous vein bypass grafts. Histochem Cell Biol 2013;140:183-8.

43. Chang L, Karin M. Mammalian MAP kinase signalling cascades. Nature 2001;410:37-40. 
Supplementary

Table S1 Data quantifications

\begin{tabular}{|c|c|c|c|c|}
\hline Items & \multicolumn{3}{|c|}{ Groups } & Power $(1-\beta)$ \\
\hline \multicolumn{5}{|l|}{ Diameter (mm) } \\
\hline $4 w$ & $1.94 \pm 0.09$ & $1.72 \pm 0.03$ & $1.38 \pm 0.07$ & 1 \\
\hline $8 w$ & $2.13 \pm 0.07$ & $1.58 \pm 0.05$ & $1.25 \pm 0.09$ & 1 \\
\hline $4 w$ & $671.6 \pm 32.64$ & $780.2 \pm 93.52$ & $884.4 \pm 71.5$ & 1 \\
\hline $8 w$ & $633 \pm 51.2$ & $761.8 \pm 113.78$ & $816.8 \pm 161.54$ & 0.9999964 \\
\hline \multicolumn{5}{|l|}{ Wall thickness (mm) } \\
\hline $4 w$ & $129.06 \pm 23.2$ & $89.94 \pm 14.76$ & $57.17 \pm 12.91$ & 0.9996988 \\
\hline $4 w$ & $0.78 \pm 0.07$ & $0.63 \pm 0.05$ & $0.3 \pm 0.05$ & 1 \\
\hline $8 w$ & $1.02 \pm 0.21$ & $0.74 \pm 0.13$ & $0.32 \pm 0.07$ & 0.9999497 \\
\hline Mean IOD of TNF- $\alpha$ & $0.137 \pm 0.007$ & $0.088 \pm 0.008$ & $0.046 \pm 0.005$ & 1 \\
\hline Mean IOD of JNK & $0.013 \pm 0.001$ & $0.012 \pm 0.001$ & $0.005 \pm 0.001$ & 1 \\
\hline Mean IOD of c-Jun & $0.013 \pm 0.002$ & $0.007 \pm 0.001$ & $0.003 \pm 0.001$ & 1 \\
\hline TUNEL+ cells & $37.45 \pm 7.45$ & $16.33 \pm 4.3$ & $6.42 \pm 2.21$ & 1 \\
\hline
\end{tabular}

Data were presented as mean \pm SD flowed by one-way ANOVA analysis. Statistical significances were represented on the relevant figures. 